\title{
Physical and structural studies of chemical Vapor deposited lacunar neodymium manganite thin films
}

\author{
N. Ihzaz a ${ }^{1,2}$, M. Boudard ${ }^{3}$, L. Rapenne ${ }^{3}$, H. Roussel $^{3}$, S. Pignard ${ }^{3}$, and M. Oumezzine ${ }^{1}$ \\ ${ }^{1}$ Laboratoire de Physico-Chimie des Matériaux, Département de Physique, Faculté des Sciences de \\ Monastir, 5019 Monastir, Tunisia \\ 2 Institut Supérieur des Sciences Appliquées et de Technologie de Gabès, Rue Amor Ben El Khatab, \\ 6029 Gabès, Tunisia \\ ${ }^{3}$ LMGP, MINATEC Bâtiment INPG, UMR 5628 CNRS/INPG, 3 Parvis Louis Néel, BP 257, 38016 \\ Grenoble Cedex 1, France
}

\begin{abstract}
Lacunar $\mathrm{Nd}_{1-\mathrm{x}} \mathrm{MnO}_{3-\delta}$ thin films were successfully deposited by a liquid source metal-organic MOCVD technique on (001) $\mathrm{SrTiO}_{3}$ (STO), $\mathrm{LaAlO}_{3}(\mathrm{LAO})$ and $\mathrm{Si}$ substrates. Optimal growth conditions are reported. TEM and X-ray diffraction characterisations reveal that the thin films grown on STO and LAO substrates are single crystalline layers epitaxially grown on the top of the substrates. The relationship between the crystallographic orientation of the films and those of the substrates were determined. Magnetic transition temperatures (Tc) of the as-grown STO films, deduced from Squid Magnetometer measurements, are compared to the corresponding bulk values (typically 100K). Our magnetic measurements also suggest a complicated magnetic behavior close to the one observed in bulk samples: magnetization curves obtained under different applied magnetic fields indicate a possible reverse of the magnetization sign at low temperatures.
\end{abstract}

Keywords: CVD, thin film, X-ray diffraction, magnetic properties.

PACS: 73-43-Qt. 75-50-Ee.

\section{Introduction}

Neodymium manganese oxides with the general formula $\mathrm{Nd}_{1-\mathrm{x}} \mathrm{A}_{\mathrm{x}} \mathrm{MnO}_{3}$, where $\mathrm{A}$ is a divalent alkaline-earth, monovalent alkali metal or represents a deficiency in $\mathrm{Nd}$ content, have been extensively investigated because of their unexpected magnetic phenomena which is a considerable subject in scientific studies due to its potential application [1]. Depending on the type and amount of doping, these materials exhibit a variety of phenomena, such as double-exchange (DE), antiferromagnetic super-exchange (SE) interactions between $\mathrm{Mn}^{3+}$ and $\mathrm{Mn}^{4+}$ ions and phase segregation [2-5]. More attention has been focused on a certain class of manganese perovskites belonging to the group of orthorhombic distorted perovskites $\mathrm{Nd}_{1-\mathrm{x}} \mathrm{MnO}_{3}$. In orthorhombic perovskites there is a strong competition between DE and SE interactions of Mn ions; as a result a complex magnetic structure is established. If, beside $\mathrm{d}-\mathrm{d}$ interactions of manganese ions, $\mathrm{f}-\mathrm{d}$ and $\mathrm{f}-\mathrm{f}$ interactions of rare-earth ions exist, the magnetic behavior of $\mathrm{Nd}_{1-\mathrm{x}} \mathrm{MnO}_{3}$ perovskites becomes much more complicated [6-8]. In fact the so called $\mathrm{NdMnO}_{3}$ is a much more complex material than

\footnotetext{
a e-mail : Nejib.ihzaz@issatgb.rnu.tn
} 
indicated by this simple nominal composition, because both the $\mathrm{Nd} / \mathrm{Mn}$ ratio and the oxygen content appear to be variable [9] and the presence of holes on cation and oxygen sites plays an important role in magnetic and transport properties. So the aim of this paper was to prepare a lacunar-controlled neodymium manganite thin films in order to make a comparison study with the bulk component.

\section{Experimental}

$\mathrm{NdMnO}_{3}$ (hereafter NMO) thin films were synthesized by metal-organic MOCVD molecular injection. A detailed description of this experimental technique has been presented in Ref. [10]. The scheme of the horizontal shaped CVD reactor which has been used for the synthesis of NMO films is presented in Fig. 1. The particular characteristic of this MOCVD technique is the sequential injection of micro-amounts of a liquid solution of solid precursors diluted in a solvent [11]. These droplets of a few $\mathrm{mm}^{3}$ are injected in a cold zone on a moving belt at a working pressure of a few hundreds $\mathrm{Pa}$, the solvent is immediately evaporated and in-situ eliminated. The precise micro-amount of solid precursor lying on the moving belt is mechanically carried into an evaporation zone where the precursor's vapours are produced. These vapours are then carried into the reaction zone where a classical CVD reaction occurs on a substrate. The flow rate and the concentration of precursors in the evaporator depend on the aperture time, the injection frequency, the viscosity of the solution of precursors and on the pressurization of the vessel containing the solution. NMO layers were obtained from a solution of 2,2,6,6 tetramethyl 3,5 heptanedionato- neodymium and manganese (i.e. $\mathrm{Nd}(\mathrm{tmhd})_{3}$ and $\mathrm{Mn}(\mathrm{tmhd})_{3}$ ) dissolved in 1,2 dimethoxyethane solvent at a total concentration of 0.02 $\mathrm{mol} \mathrm{l}^{-1}$. As the ratio of each precursor in solution is generally different from the concentration ratio obtained in the deposited film, we have first studied each precursor for measuring its deposition rate versus the composition of the solution and the deposition temperature. Metal-organic vapours were transported in a carrier gas flow of $600 \mathrm{~cm}^{3} \mathrm{~min}^{-1}$, constituted of $\mathrm{O}_{2}(50 \%)$ and $\mathrm{Ar}(50 \%)$ under a total pressure of 10 mbar (optimized conditions are summarized in Table 1). After the deposition films are ex-situ annealed at the same temperature $\left(680{ }^{\circ} \mathrm{C}\right)$ for 30 min under an $\mathrm{O}_{2}$ flow and as a second step the film was annealed at $900^{\circ} \mathrm{C}$ for 24 hours in air. Film compositions were checked by energy dispersive X-ray spectroscopy analysis (EDX). Phase purity and crystallization were studied by X-ray diffraction (hereafter XRD) in Bragg-Brentano geometry using a BRUKER D8 advance diffractometer with monochromatic $\mathrm{CuK \alpha} 1$ radiation $\lambda=0.154060 \mathrm{~nm}$ and LynxEye 1 dimension detector.

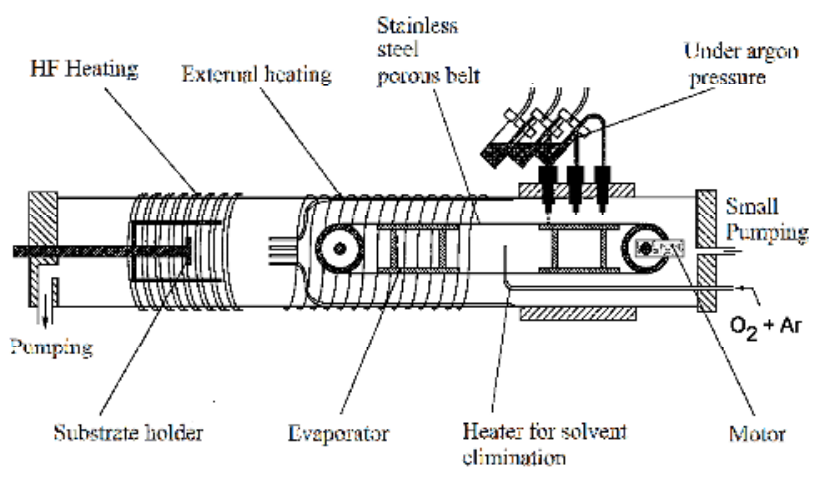

Fig. 1. Schema of the CVD reactor

Transmission electron microscopy (TEM) observations were carried out at $200 \mathrm{kV}$ with a JEOL 2010 microscope $(0.19 \mathrm{~nm}$ resolution).

Magnetization (M) vs. temperature (T) were measured using squid magnetometers (Quantum Design) with generally applied magnetic field of 500 Oe in field cooled (FC) and zero field cooled (ZFC) regimes in 10 - $140 \mathrm{~K}$ temperature range. 
Table 1. Growth conditions

\begin{tabular}{ll}
\hline Parameter & Value \\
\hline $\mathrm{Nd}(\text { tmhd })_{3}$ dilution & $2.10^{-2} \mathrm{~mol} / \mathrm{l}$ \\
$\mathrm{Mn}(\mathrm{tmhd})_{3}$ dilution & $2.10^{-2} \mathrm{~mol} / \mathrm{l}$ \\
Injection temperature & $30^{\circ} \mathrm{C}$ \\
Evaporation temperature & $250^{\circ} \mathrm{C}$ \\
Substrate temperature & $680^{\circ} \mathrm{C}$ \\
Injection frequency & $1 \mathrm{~Hz}$ \\
Total pressure & $10 \mathrm{mbar}$ \\
$\mathrm{O}_{2}$ flow & $600 \mathrm{~cm}^{3} / \mathrm{min}$ \\
Ar flow & $600 \mathrm{~cm}^{3} / \mathrm{min}$ \\
first annealing & $30 \mathrm{~min}, 1 \mathrm{bar}, 680^{\circ} \mathrm{C}, \mathrm{O}_{2}$ flow: \\
second annealing & $600 \mathrm{~cm} / \mathrm{min}$ \\
& $24 \mathrm{hours}, 1 \mathrm{bar}, 900^{\circ} \mathrm{C}$ in air \\
\hline
\end{tabular}

\section{Results and discussion}

We report on preliminary results on the growth and characterization of NMO perovskite thin films grown under epitaxial strain $(\mathrm{Nd} / \mathrm{Mn}$ ratio $(\mathrm{EDX})=0.88)$.

Fig. 2 (a) shows that as-deposited NMO layer is amorphous since no peaks apart from the $\mathrm{SrTiO}_{3}$ (001) (hereafter STO) substrate reflections are visible. Moreover, an explicit amorphous hump is established. In fact, the first stage of deposition at $680{ }^{\circ} \mathrm{C}$ does not introduce any significant change in the XRD pattern, indicating that NMO layer remains amorphous after this processing step. After annealing at $900{ }^{\circ} \mathrm{C}$ for 24 hours, clear diffraction peaks appear (Fig. 2 b).
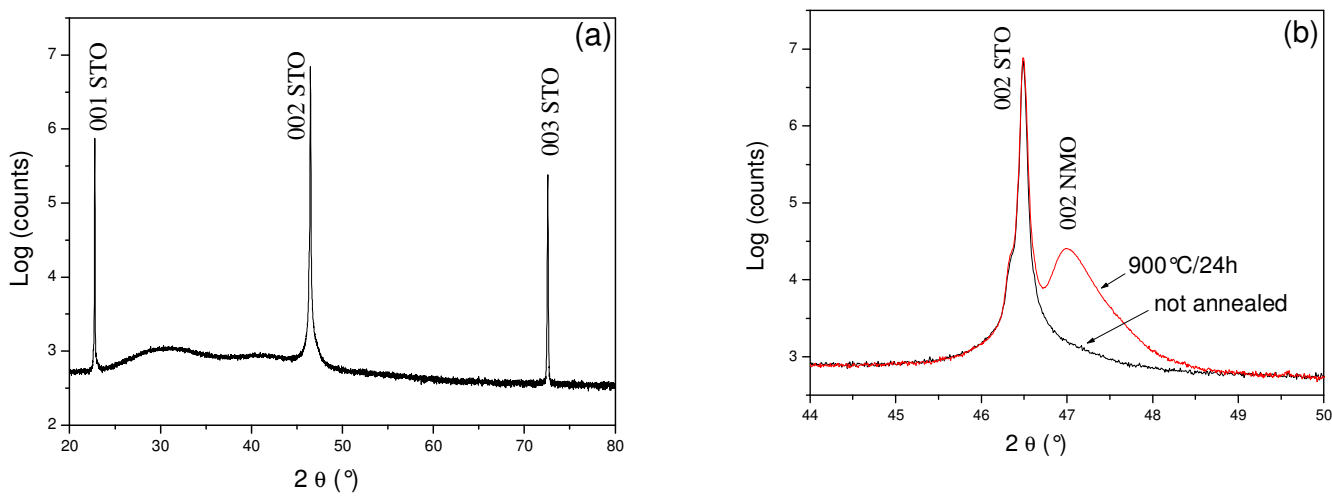

Fig. 2. X-ray diffraction pattern of NMO/STO films. MOCVD as grown films with two different growing conditions. (a): annealed at $680^{\circ} \mathrm{C}$ for half of an hour. (b): subsequently annealed at $900^{\circ} \mathrm{C}$ for 24 hours

Bragg reflections $h O h$ of the thin films are clearly seen nearby $00 l$ reflections of the substrate. Furthermore, the sharpness of the diffraction peaks of the NMO thin film with 200nm in thickness film and the fact that the $h 0 h$ Bragg reflection almost coincide with $00 l$ Bragg peaks of STO $\left(c_{\mathrm{STO}}=0.3905 \mathrm{~nm}\right)$ indicates that this film is epitaxially grown on the substrate with similar orientation relationship that given or studied in the previous works [12,13]. This means that the NMO thin film with $200 \mathrm{~nm}$ in thickness grown on the STO substrate presents a fairly good crystallinity and a strong in-plane texture with the [101]-axis perpendicular to the surface of the substrate. No other peaks are visible, suggesting a single orientation and the absence of second-phase precipitatation. The film pseudo-cubic cell parameter, perpendicular to the surface of the substrate, 
which can be deduced from the scan are $0.3863 \mathrm{~nm}(\mathrm{Nd} / \mathrm{Mn}=0.88)$. The lattice spacing of STO $\left(c_{\mathrm{STO}}\right.$ $=0.3905 \mathrm{~nm}$ ) being larger than the lattice spacing of NMO deduced from XRD scan, the layer is subjected to in-plane tensile strain which should give rise to a contraction of the layer out-of-plane lattice spacing.

In the aim to provide comparative study, thin film with $150 \mathrm{~nm}$ in $(\mathrm{Nd} / \mathrm{Mn}=0.96)$ was grown on STO (001) substrate, and characterized by XRD before annealing at $900^{\circ} \mathrm{C}$ (see left part in Fig.3). According to high resolution electron images of NMO thin film with $150 \mathrm{~nm}$ in thickness $(\mathrm{Nd} / \mathrm{Mn}=$ 0.96) presented in Fig.3, well-defined orientation relationships at the vicinity of the interface between film and substrate can be identified as: $[010]_{\mathrm{NMO}} / /[010]_{\mathrm{STO}}$ and $[101]_{\mathrm{NMO}} / /[001]_{\mathrm{STO}}$. Growth morphology of the MOCVD as grown film can be roughly described as a coexistence of both epitaxial crystalline region near the interface with the substrate and large amorphous regions at the top part of the film. Subsequent annealing at $900{ }^{\circ} \mathrm{C}$ for 24 hours improves the quality of the film as results in sharp diffraction peaks as can be seen in the Fig. 2 (b).
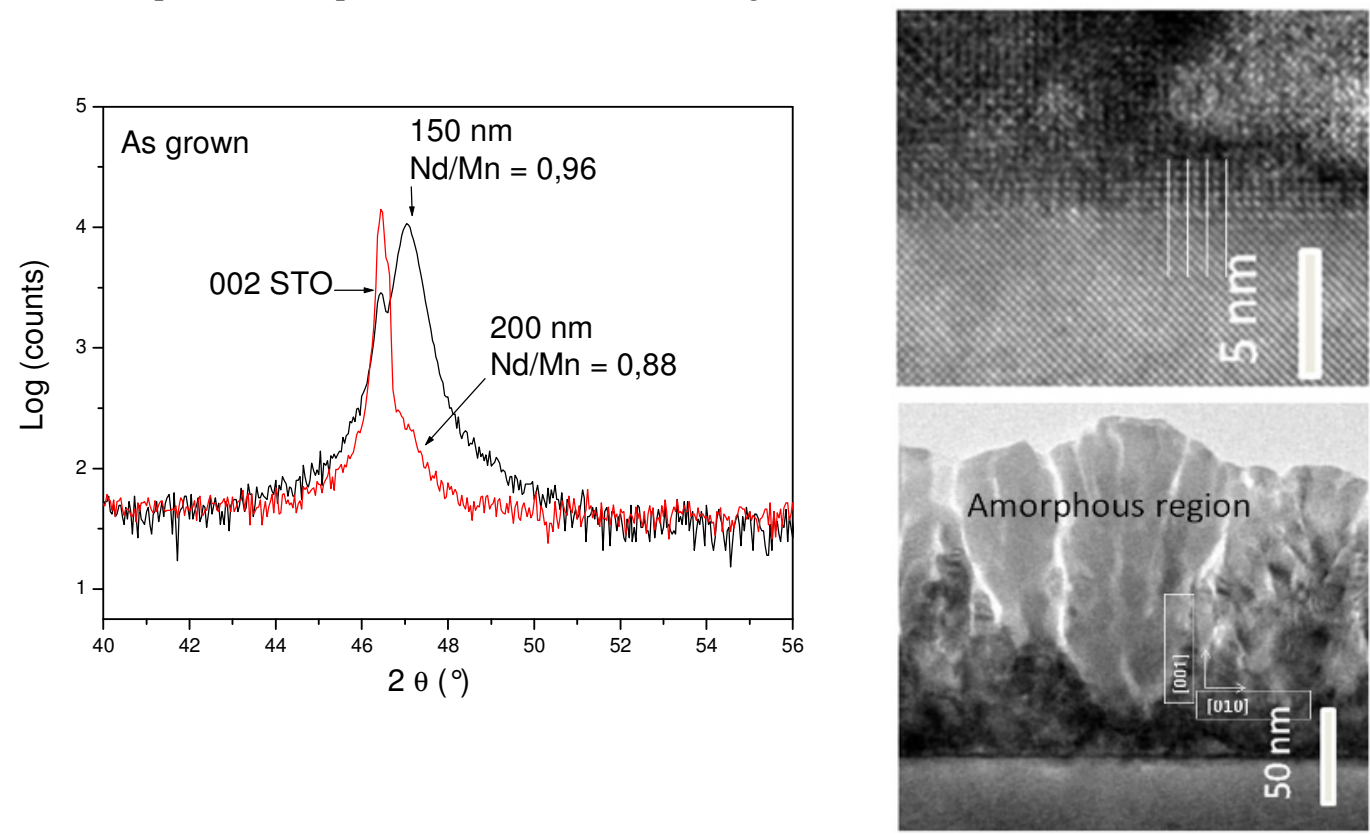

Fig. 3. Left part: X-ray diffraction pattern of NMO/STO for both films $(\mathrm{Nd} / \mathrm{Mn}=0.96$ and $\mathrm{Nd} / \mathrm{Mn}=$ 0.88 ) without annealing at $900^{\circ} \mathrm{C}$. Right part: TEM observations on the as grown thin film with $200 \mathrm{~nm}$ in thickness (Nd/Mn ratio of 0.96). Top: Part of the film is epitaxially grown on the STO substrate with $[010]_{\mathrm{NMO}} / /[010]_{\mathrm{STO}}$ and $[101]_{\mathrm{NMO}} / /[001]_{\mathrm{STO}}$. White lines indicate $b_{\text {orth }}$ cell parameter. Bottom: large amorphous regions are present on the top part of the film

Fig.4 shows the FC curve of magnetization versus temperature during warming up from 10 to $150 \mathrm{~K}$ measured under an applied magnetic field of 500 Oe for the bulk material. FC curve of the film case is reproduced in the inset. Magnetic transition at almost $100 \mathrm{~K}$ is clearly present. 


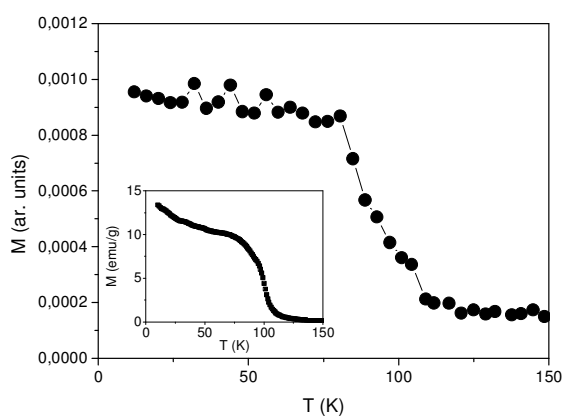

Fig. 4. FC spontaneous magnetization curves measured under 500 Oe field for bulk material. Inset: FC magnetization measured under an applied magnetic field of 500 Oe for the thin film

Indeed Fig.5 , shows that both ZFC and FC curves at 500 Oe are similar whatever the sample is in the form of an annealed film (left part of the figure) or in the form of ceramic powder samples [7] with similar composition (right part of the figure).

Moreover, a cusp in the ZFC curve for both film and bulk compound, as a function of temperature collected at 500 Oe can be clearly seen. A distinctive separation of the FC and ZFC curves may be explained by a cluster (spin) glass-like transition. This feature is in marked contrast to that found in the A-type manganite.

Negative magnetization that was observed in curve of FC magnetization versus temperature at 200 $\mathrm{Oe}$ and $50 \mathrm{Oe}$ may be explained in term of $\mathrm{Nd}$ polarization in the direction opposite to that of the $\mathrm{Mn}^{3+}$ magnetic moments because of the strong f-d interactions for $\mathrm{NdMnO}_{3}$. Related to our studied sample Troyanchuck et al. [14] suggest that an interpretation based on a coexistence of AFM and FM phases as for $\mathrm{Nd}_{1-x} \mathrm{Ca}_{x} \mathrm{MnO}_{3}$ samples [15] stands for $\mathrm{Nd}_{0.9} \mathrm{MnO}_{x}$ samples. According to their hypothesis the $\mathrm{f}-\mathrm{d}$ exchange between $\mathrm{Mn}$ and $\mathrm{Nd}$ ions is negative in the AFM phase (and thus similar to their interpretation of $\mathrm{NdMnO}_{3}$ ) and positive in the FM phase at high temperature. At low temperature the exchange interaction is positive for both phases i.e. $\mathrm{Nd}$ and FM Mn component are in the same direction for both phases. Furthermore Bartolome et al. [16] show additional evidence that $\mathrm{Nd}$ and $\mathrm{Mn}$ net magnetization are parallel along a large range of temperature ruling out the competition between $\mathrm{Nd}$ and $\mathrm{Mn}$ sub-lattices as the origin of the negative magnetization observed in $\mathrm{NdMnO}_{3.11}$ and related systems.
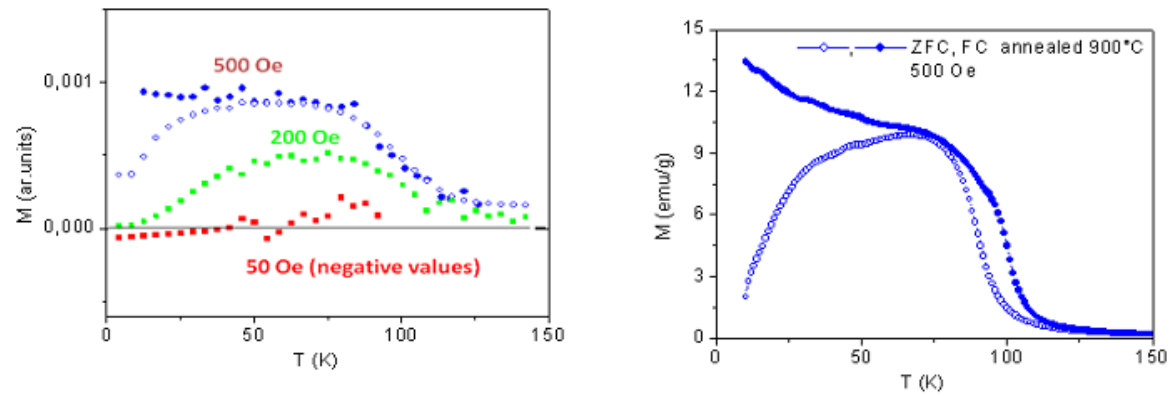

Fig. 5. Magnetic measurements. Left: Squid magnetometer results on the annealed film at $900{ }^{\circ} \mathrm{C}$ for 24 hours (sample thickness $200 \mathrm{~nm} \mathrm{Nd} / \mathrm{Mn}$ ratio $(\mathrm{EDX})=0.88$ ). Right: $\mathrm{ZFC}, \mathrm{FC}$ curves for ceramic powder samples with similar composition [7]. 


\section{Conclusion}

In summary, we have succeeded in the stabilization of epitaxial films of NMO on STO (001) substrates by an MOCVD process. These films present a high crystalline quality. From TEM and XRD experiments, structural quality of NMO thin films has been determined before and after annealing at $900^{\circ} \mathrm{C}$ for 24 hours. Preliminary results on NMO show that magnetic properties of annealed thin film are similar to those of bulk sample.

\section{References}

1. E. Dagotto, T. Hotta, A. Moreo, Phys. Rep. 344, 1-3 (2001)

2. C. Zener, Phys. Rev. 82, 403-407 (1951)

3. P.W. Anderson, H. Hasegawa, Phys. Rev. 100, 675-680 (1955)

4. P. G. De Gennes, Phys. Rev. 118, 14-145 (1960)

5. J. B. Goodenough, Prog. Solid State Chem. 5149 (1971)

6. A. Munoz, J. A. Alonso, M. J. Martinez-Lope, J. L. Garcia-Munoz, M. T. Fernandez-Diaz, J. Phys.: Condens. Matter 12, 1361 (2000)

7. N. Ihzaz, M. Boudard, H. Vincent, M. Oumezzine, Journal of Alloys and Compounds, 479, 445-450 (2009)

8. F. Bartolomé, J. Bartolomé, J. Campoa, Physica B 312, 769-771(2002)

9. E. T. Maguire, A M. Coats, J. M. S. Skakle, A. R. West, J.Mater. Chem.9 1337 (1999)

10. S. Pignard, H. Vincent, J. P. Sénateur, P. H. Giauque, Thin Solid Films 347 ,161 (1999)

11. J. P. Sénateur, R. Madar, F. Weiss, O. Thomas and A. Abrutis, French Patent 2707671 (1993) Eur. Patent 730671 (1994), US patent 945162 (1997)

12. C. Girardot, F. Conchon, A. Boulle, P. Chaudouet, N. Caillault, J. Kreisel, R. Guinebreti ere, F. Weiss, S. Pignard, Surface and Coatings Technology 201, $9021-9024$ (2007). Euro CVD 16, 16th European Conference on Chemical Vapor Deposition.

13. N. Ihzaz, S. Pignard, J. Kreisel, H. Vincent, J. Marcus, J. Dhahri, M. Oumezzine, Physica status solidi (c) 1, 1679-1682 (2004)

14. I. O. Troyanchuk, N. V. Pushkarev, M. V. Bushinskii, E. Gamari-Seale, Phys. Solid State 48 $1315(2006)$

15. H. Gamari-Seale, I.O. Troyanchuk,D. Khalyalin, H. Szymczak, K.L. Stefanopoulos, J. Hernandez-Velasco, J. Magn. Magn. Mater. 272 e483 (2004)

16. F. Bartolome, J. Herrero-Albillos, L. M. Garcia, J. Bartolome, N. Jaouen, A. Rogalev, J. Appl. Phys. 97 (10) A503 (2005) 